\title{
On Some Existence and Uniqueness Results for a Class of Equations of Order $0<\alpha \leq 1$ on Arbitrary Time Scales
}

\author{
Abdourazek Souahi, ${ }^{1}$ Assia Guezane-Lakoud, ${ }^{2}$ and Rabah Khaldi ${ }^{2}$ \\ ${ }^{1}$ Laboratory of Applied Mathematics and Modeling, University of 8 May 1945 Guelma, P.O. Box 401, 24000 Guelma, Algeria \\ ${ }^{2}$ Laboratory of Advanced Materials, University of Badji Mokhtar-Annaba, P.O. Box 12, 23000 Annaba, Algeria
}

Correspondence should be addressed to Abdourazek Souahi; arsouahi@yahoo.fr

Received 21 April 2016; Accepted 9 June 2016

Academic Editor: Patricia J. Y. Wong

Copyright (C) 2016 Abdourazek Souahi et al. This is an open access article distributed under the Creative Commons Attribution License, which permits unrestricted use, distribution, and reproduction in any medium, provided the original work is properly cited.

This paper investigates the existence and uniqueness of solution for a class of nonlinear fractional differential equations of fractional order $0<\alpha \leq 1$ in arbitrary time scales. The results are established using extensions of Krasnoselskii-Krein, Rogers, and Kooi conditions.

\section{Introduction}

This work concerns the investigation of sufficient conditions for the existence and uniqueness of the solution of the following initial value problem with fractional derivative up to the first order on arbitrary time scales:

$$
\begin{aligned}
& { }_{t}^{\pi} D^{\alpha} u(t)=f(t, u(t)), \\
& t \in\left[t_{0}, t_{0}+a\right]_{\mathbb{T}}, 0<\alpha \leq 1,
\end{aligned}
$$

$$
{ }_{t}^{\pi} I^{1-\alpha} u\left(t_{0}\right)=0,
$$

where ${ }_{t}^{\pi} D^{\alpha}$ is the (left) Riemann-Liouville fractional derivative of order $\alpha$ on time scales $\mathbb{T}$, ${ }_{t}^{\mathbb{T}} I$ is the Riemann-Liouville fractional integral on time scales, and $\left[t_{0}, t_{0}+a\right]_{\mathbb{T}}$ is an interval on $\mathbb{T}$. We assume that $f$ is a right-dense continuous function.

The theory of time scales calculus allows us to study the dynamic equations, which include both difference and differential equations, both of which are very important in implementing applications; for further information about the theoretical and potential applications of the theory of time scales, we refer the reader to [1-8] and the survey [9].

The quantitative behaviour of solutions to ordinary differential equations on time scales is currently undergoing active investigations. Many authors studied the existence and the uniqueness of the solutions of initial and boundary differential equations; see $[8,10-20]$ and the references cited therein. In the papers [21-25], several authors were interested by the existence and uniqueness of the first-order differential equations on time scales with initial or boundary conditions using diverse techniques and conditions. On the other hand, some existence results for the fractional order differential equations were obtained in [10].

Our ideas arise from the papers [26-34], especially [30, 31], where the authors used Nagumo and Krasnoselskii-Krein conditions on the nonlinear term $f$, without satisfying Lipschitz assumption. Motivated greatly by the above works, under appropriate time scales versions of the KrasnoselskiiKrein conditions, we obtain the uniqueness and existence of solution for the following two classes of differential equations, namely, the first-order ODE

$$
\begin{aligned}
& u^{\Delta}(t)=f(t, u(t)), \quad t \in\left[t_{0}, t_{0}+a\right]_{\mathbb{T}}, \\
& u\left(t_{0}\right)=0,
\end{aligned}
$$

and the fractional order FDE:

$$
\begin{aligned}
& { }_{t}^{\pi} D^{\alpha} u(t)=f(t, u(t)), \\
& t \in\left[t_{0}, t_{0}+a\right]_{\mathbb{T}}, 0<\alpha \leq 1,
\end{aligned}
$$

$$
{ }_{t}^{\mathbb{T}} I^{1-\alpha} u\left(t_{0}\right)=0
$$


The rest of the paper is organized as follows. In Section 2, we give some definitions and lemmas that will be used in our work. Section 3 is devoted to the main results; we first establish the uniqueness of the solution under Krasnoselskii-Krein conditions for the first-order problem; then we establish the convergence of the successive approximations to the unique solution. Later, we prove the uniqueness for the fractional order problem under some other conditions.

\section{Preliminaries}

In this section, we recall basic results and definitions in time scales calculus.

A time scale $\mathbb{T}$ is a nonempty closed subset of $\mathbb{R}$. We assume that $\operatorname{card}(\mathbb{T}) \geq 2$. The forward and backward jump operators $\sigma, \rho: \mathbb{T} \rightarrow \mathbb{T}$, are, respectively, defined by

$$
\begin{aligned}
& \sigma(t)=\inf \{s \in \mathbb{T}: s>t\}, \\
& \rho(t)=\sup \{s \in \mathbb{T}: s<t\} .
\end{aligned}
$$

The point $t \in \mathbb{T}$ is left-dense, left-scattered, right-dense, and right scattered if $\rho(t)=t, \rho(t)<t, \sigma(t)=t$, and $\sigma(t)>t$, respectively.

We set $\mathbb{T}^{\mathscr{K}}=\mathbb{T} \backslash\{\max \mathbb{T}\}$ whenever $\mathbb{T}$ admits a left-scattered maximum, and $\mathbb{T}^{\mathscr{K}}=\mathbb{T}$ otherwise. We denote $A_{\mathbb{T}}=$ $A \cap \mathbb{T}$. An interval of $\mathbb{T}$ is defined by $I_{\mathbb{T}}$, where $I$ is an interval of $\mathbb{R}$.

Definition 1 (delta derivative [1]). Assume $f: \mathbb{T} \rightarrow \mathbb{R}$ and let $t \in \mathbb{T}^{\mathscr{K}}$. We define

$$
f^{\Delta}(t)=\lim _{s \rightarrow t} \frac{f(\sigma(s))-f(t)}{\sigma(s)-t}, \quad t \neq \sigma(s),
$$

provided the limit exists. We call $f^{\Delta}(t)$ the delta derivative (or Hilger derivative) of $f$ at $t$. Moreover, we say that $f$ is delta differentiable on $\mathbb{T}^{\mathscr{K}}$ provided $f^{\Delta}$ exists for all $t \in \mathbb{T}^{\mathscr{K}}$. The function $f^{\Delta}: \mathbb{T}^{\mathscr{K}} \rightarrow \mathbb{R}$ is called the (delta) derivative of $f$ on $\mathbb{T}^{\mathscr{K}}$.

Definition 2 (see [10]). A function $f: \mathbb{T} \rightarrow \mathbb{R}$ is called rdcontinuous provided it is continuous at right-dense points in $\mathbb{T}$ and its left-sided limits exist (finite) at left-dense points in $\mathbb{T}$. The set of rd-continuous function $f: \mathbb{T} \rightarrow \mathbb{R}$ is denoted by $\mathscr{C}_{\text {rd }}$. Similarly, a function $f: \mathbb{T} \rightarrow \mathbb{R}$ is called ld-continuous provided it is continuous at left-dense points in $\mathbb{T}$ and its right-sided limits exist (finite) at right-dense points in $\mathbb{T}$. The set of ld-continuous function $f: \mathbb{T} \rightarrow \mathbb{R}$ is denoted by $\mathscr{C}_{\mathrm{ld}}$. For $f \in \mathscr{C}_{\text {rd }}$ define $\|f\|=\sup _{t \in \mathbb{T}}|f(t)|$. It is easy to see that $\mathscr{C}_{\text {rd }}$ is a Banach space with this norm.

Definition 3 (delta antiderivative [10]). A function $F$ : [a, $b]_{\mathbb{V}} \rightarrow \mathbb{R}$ is called a delta antiderivative of a function $f:$ $[a, b)_{\mathbb{T}} \rightarrow \mathbb{R}$ provided $F$ is continuous on $[a, b]_{\mathbb{T}}$, delta differentiable on $[a, b)_{\mathbb{T}}$, and $F^{\delta}(t)=f(t)$ for all $t \in[a, b)_{\mathbb{T}}$. Then, we define the $\Delta$-integral of $f$ from $a$ to $b$ by

$$
\int_{a}^{b} f(t) \Delta t \triangleq F(b)-F(a) .
$$

Lemma 4. Let $f$ be an increasing continuous function on the $[a, b]_{\mathbb{T}}$. We define the extension $\tilde{f}$ of $f$ to the real interval $[a, b]$ by

$$
\widetilde{f}(s) \triangleq \begin{cases}f(s) & \text { if } s \in \mathbb{T}, \\ 0 & \text { if } s \in(t, \sigma(t)) \notin \mathbb{T} .\end{cases}
$$

Then

$$
\begin{aligned}
\int_{a}^{b} f(t) \Delta t & \leq \int_{a}^{b} \tilde{f}(t) d t, \\
\tilde{f}^{\Delta}(t) & =f^{\Delta}(t), \quad \text { for every } t \in(a, b)_{\mathbb{T}} .
\end{aligned}
$$

Lemma 5. Let $y:\left[t_{0}, t_{0}+a\right]_{\mathbb{T}} \rightarrow \mathbb{R}$ be continuous. Then the general solution of the differential equation

$$
u^{\Delta}(t)=y(t)
$$

is given by

$$
u(t)=u\left(t_{0}\right)+\int_{t_{0}}^{t} y(s) \Delta s, \quad t \in\left[t_{0}, t_{0}+a\right]_{\mathbb{T}} .
$$

Proof. Lemma 5 is an immediate consequence of Theorem 4.1 [5].

Definition 6 (fractional integral on time scales [10]). Suppose $\mathbb{T}$ is a time scale, $[a, b]$ is an interval of $\mathbb{T}$, and $h$ is an integrable function on $[a, b]$. Let $0<\alpha<1$. Then the (left) fractional integral of order $\alpha$ of $h$ is defined by

$$
{ }_{a}^{\mathbb{T}} I_{t}^{\alpha} h(t)=\int_{a}^{t} \frac{(t-s)^{\alpha-1}}{\Gamma(\alpha)} h(s) \Delta s,
$$

where $\Gamma$ is the gamma function.

Definition 7 (fractional Riemann-Liouville derivative on time scales [10]). Let $\mathbb{T}$ be a time scale, $t \in \mathbb{T}, 0<\alpha<1$, and $h$ : $\mathbb{T} \rightarrow \mathbb{R}$. Then the (left) Riemann-Liouville fractional derivative of order $\alpha$ of $h$ is defined by

$$
{ }_{a}^{\mathbb{T}} D_{t}^{\alpha} h(t)=\frac{1}{\Gamma(1-\alpha)}\left(\int_{a}^{t}(t-s)^{-\alpha} h(s) \Delta s\right)^{\Delta} .
$$

For the sake of simplicity, we use the following notation ${ }_{t}^{\mathbb{T}} I^{\alpha}$ and ${ }_{t}^{\mathbb{T}} D^{\alpha}$ instead of ${ }_{t_{0}}^{\mathbb{T}} I_{t}^{\alpha}$ and ${ }_{t_{0}}^{\mathbb{T}} D_{t}^{\alpha}$, respectively, whenever $a=t_{0}$.

Lemma 8 (see $[10])$. For any function $f$ integrable on $\left[t_{0}, t_{0}+\right.$ a $]_{\mathbb{T}}$ one has the following:

$$
\left({ }_{t}^{\mathbb{T}} D^{\alpha} \circ{ }_{t}^{\mathbb{T}} I^{\alpha}\right)(f)=f .
$$

Lemma 9 (see [10]). Let $f \in C\left(\left[t_{0}, t_{0}+a\right]_{\mathbb{V}}\right)$ and $0<\alpha<1$. If $\left.{ }_{t}^{\pi} I^{1-\alpha} f(t)\right|_{t=t_{0}}=0$, then

$$
\left({ }_{t}^{\mathbb{T}} I^{\alpha} \circ{ }_{t}^{\mathbb{T}} D^{\alpha}\right)(f)=f \text {. }
$$


Lemma 10 (see [10]). Let $0<\alpha<1$ and $f:\left[t_{0}, t_{0}+a\right]_{\mathbb{T}} \times \mathbb{R} \rightarrow$ $\mathbb{R}$. The function $u$ is a solution of problem (2) if and only if it is a solution of the following integral equation:

$$
\begin{aligned}
u(t)=\frac{1}{\Gamma(\alpha)} \int_{t_{0}}^{t}(t-s)^{\alpha-1} f(s, u(s)) \Delta s, & \\
t & \in\left[t_{0}, t_{0}+a\right]_{\mathbb{T}} .
\end{aligned}
$$

Lemma 11 (see [31]). The solution of the equation

$$
{ }_{R L} D_{t_{0}}^{\alpha} R(t)=[R(t)]^{\delta}
$$

is given by

$$
R(t)=L\left(t-t_{0}\right)^{\sigma},
$$

where $L=(\Gamma(1-\alpha))^{1 /(1-\delta)}$ and $\sigma=\alpha /(1-\delta)$ and ${ }_{R L} D_{t_{0}}^{\alpha}$ is the fractional Riemann-Liouville derivative of order $\alpha \in(0,1)$ on the interval $\left[t_{0}, t_{0}+a\right]$; see [35].

\section{Main Results}

In the following, we denote $S_{0}=\left\{(t, x): t \in\left[t_{0}, t_{0}+a\right]_{\mathbb{T}}\right.$, $\left.|x| \leq b, a, b \in \mathbb{R}^{+}\right\}$.

\subsection{Uniqueness Results for First-Order ODE}

Theorem 12 (Krasnoselskii-Krein conditions). Let $f(t, x)$ be continuous in $S_{0}$ and for all $(t, x),(t, \bar{x}) \in S_{0}$ satisfying

(H1) $|f(t, x)-f(t, \bar{x})| \leq k\left|t-t_{0}\right|^{-1}|x-\bar{x}|, t \neq t_{0}$,

(H2) $|f(t, x)-f(t, \bar{x})| \leq c|x-\bar{x}|^{\delta}$, where $c$ and $k$ are positive constants; the real number $\delta$ is such that $0<\delta<1$, and $k(1-\delta)<1$.

Then, the first-order initial value problem (2) has at most one solution on $\left[t_{0}, t_{0}+a\right]_{\mathbb{T}}$.

Proof. Suppose $u$ and $v$ are two solutions of (2) in $\left[t_{0}, t_{0}+a\right]_{\mathbb{T}}$. We will show that $u \equiv v$. Let us define $\phi(t)$ and $R(t)$ by

$$
\begin{aligned}
& \phi(t)=|u(t)-v(t)|, \quad \text { for every } t \in\left[t_{0}, t_{0}+a\right]_{\mathbb{T}}, \\
& R(t)=\int_{t_{0}}^{t} c \widetilde{\phi}^{\delta}(s) d s, \quad \text { for every } t \in\left[t_{0}, t_{0}+a\right],
\end{aligned}
$$

such that $\widetilde{\phi}$ is the extension of $\phi$ to the real interval $\left[t_{0}, t_{0}+a\right]$. It follows from condition (H2) that

$$
\begin{aligned}
\phi(t) & =\left|\int_{t_{0}}^{t}[f(s, u(s))-f(s, v(s))] \Delta s\right| \\
& \leq \int_{t_{0}}^{t}|f(s, u(s))-f(s, v(s))| \Delta s \\
& \leq \int_{t_{0}}^{t} c|u(s)-v(s)|^{\delta} \Delta s \leq \int_{t_{0}}^{t} c|\widetilde{u}(s)-\widetilde{v}(s)|^{\delta} d s \\
& =R(t) .
\end{aligned}
$$

On the other hand, since $R\left(t_{0}\right)=0, R(t)>0$ for $t>t_{0}$, and $R^{\Delta}(t)=c \widetilde{\phi}^{\delta}(t)$, for every $t \in\left[t_{0}, t_{0}+a\right]_{\mathbb{T}}$ we deduce from (18) and (19) that

$$
R^{\prime}(t) \leq c R^{\delta}(t), \quad \text { for every } t \in\left[t_{0}, t_{0}+a\right] .
$$

Multiplying both sides of this inequality by $(1-\delta) R^{1-\delta}(t)$ and then integrating the resulting inequality, we obtain

$$
R^{1-\delta}(t) \leq c(1-\delta)\left(t-t_{0}\right) .
$$

It immediately follows that

$$
\phi(t) \leq c^{(1-\delta)^{-1}}(1-\delta)^{(1-\delta)^{-1}}\left(t-t_{0}\right)^{(1-\delta)^{-1}} .
$$

Moreover, if we define $\psi(t)=\phi(t) /\left(t-t_{0}\right)^{k}$, we get

$$
\begin{aligned}
& 0 \leq \psi(t) \leq c^{(1-\delta)^{-1}}(1-\delta)^{(1-\delta)^{-1}}\left(t-t_{0}\right)^{(1-\delta)^{-1}-k}, \\
& \text { for every } t \in\left[t_{0}, t_{0}+a\right]_{\mathbb{T}} .
\end{aligned}
$$

It follows that the exponent of $t$ in the above inequality is positive, since $k(1-\delta)<1$. Hence, $\lim _{t \rightarrow t_{0}} \psi(t)=0$. Therefore, if we define $\psi\left(t_{0}\right)=0$, then the function is rd-continuous in $\left[t_{0}, t_{0}+a\right]_{\mathbb{T}}$.

Now, to prove that $\phi \equiv 0$, we prove by absurdity that $\psi \equiv 0$ on $\left[t_{0}, t_{0}+a\right]_{\mathbb{T}}$. Assume that $\psi$ does not vanish at some points $t$; that is, $\psi(t)>0$ on $\left.] t_{0}, t_{0}+a\right]_{\mathbb{T}}$; then there exists a maximum $m>0$ reached when $t$ is equal to some $t_{1}: t_{0}<t_{1} \leq t_{0}+a$ such that $\psi(s)<m=\psi\left(t_{1}\right)$, for $s \in\left[t_{0}, t_{1}\right)_{\mathbb{T}}$. But from condition (H1), we have

$$
\begin{aligned}
m & =\psi\left(t_{1}\right)=\left(t_{1}-t_{0}\right)^{-k} \phi\left(t_{1}\right) \\
& \leq\left(t_{1}-t_{0}\right)^{-k} \int_{t_{0}}^{t_{1}} k\left(s-t_{0}\right)^{-1} \phi(s) \Delta s \\
& \leq\left(t_{1}-t_{0}\right)^{-k} \int_{t_{0}}^{t_{1}} k\left(s-t_{0}\right)^{k-1} \psi(s) \Delta s \\
& <m\left(t_{1}-t_{0}\right)^{-k} \int_{t_{0}}^{t_{1}} k\left(s-t_{0}\right)^{k-1} \Delta s \\
& <m\left(t_{1}-t_{0}\right)^{-k} \int_{t_{0}}^{t_{1}} k\left(s-t_{0}\right)^{k-1} d s<m,
\end{aligned}
$$

which is a contradiction. Thus, the uniqueness of the solution is established.

Theorem 13 (Kooi's conditions). Let $f(t, x)$ be continuous in $S_{0}$ and satisfying for all $(t, x),(t, \bar{x}) \in S_{0}$

(I1) $|f(t, x)-f(t, \bar{x})| \leq k\left|t-t_{0}\right|^{-1}|x-\bar{x}|, t \neq t_{0}$,

(I2) $\left|t-t_{0}\right|^{\beta}|f(t, x)-f(t, \bar{x})| \leq c|x-\bar{x}|^{\delta}$, where $c$ and $k$ are positive constants; the real numbers $\beta, \delta$ are such that $0<\beta<\delta<1$, and $k(1-\delta)<1-\beta$.

Then, the first-order initial value problem (2) has at most one solution on $\left[t_{0}, t_{0}+a\right]_{\mathbb{T}}$.

Proof. The proof is similar to that of Theorem 12; thus we omit it. 


\subsection{Existence of the Solution under Krasnoselskii-Krein Conditions on Time Scales}

Theorem 14. Assume that conditions (H1) and (H2) are satisfied; then the successive approximations given by

$$
\begin{gathered}
u_{n+1}(t)=\int_{t_{0}}^{t} f\left(s, u_{n}(s)\right) \Delta s, \\
u_{0}(t)=0, \quad n=0,1, \ldots
\end{gathered}
$$

converge uniformly to the unique solution $u$ of (2) on $\left[t_{0}, t_{0}+\right.$ $\eta]_{\mathbb{T}}$, where $\eta=\min \{a, b / M\}$, and $M$ is the bound for $f$ on $S_{0}$.

Proof. With the uniqueness of the solution being proved in Theorem 12, we prove the existence of the solution using Arzela-Ascoli Theorem.

Step 1. The successive approximations $\left\{u_{n+1}\right\}, n=0,1,2, \ldots$ given by (25) are well defined and continuous. Indeed,

$$
\left|u_{n+1}(t)\right|=\left|\int_{t_{0}}^{t} f\left(s, u_{n}(s)\right) \Delta s\right| \leq \int_{t_{0}}^{t}\left|f\left(s, u_{n}(s)\right)\right| \Delta s .
$$

This yields for $n=0$

$$
\left|u_{1}(t)\right| \leq \int_{t_{0}}^{t}\left|f\left(s, u_{0}(s)\right)\right| \Delta s \leq M t \leq b .
$$

By induction, the sequence $\left\{u_{j+1}(t)\right\}$ is well defined and uniformly bounded on $\left[t_{0}, t_{0}+\eta\right]_{\mathbb{T}}$.

Step 2. We prove that $y$ is a continuous function in $\left[t_{0}, t_{0}+\eta\right]_{\mathbb{T}}$, where $y$ is defined by

$$
y(t)=\limsup _{j \rightarrow \infty}\left|u_{j}(t)-u_{j-1}(t)\right| .
$$

For $t_{1}, t_{2} \in\left[t_{0}, t_{0}+\eta\right]_{\mathbb{T}}$, we have

$$
\begin{aligned}
\left|u_{j+1}\left(t_{1}\right)-u_{j}\left(t_{1}\right)\right| \leq & \left|u_{j+1}\left(t_{2}\right)-u_{j}\left(t_{2}\right)\right| \\
& +2 M\left(t_{2}-t_{1}\right) .
\end{aligned}
$$

In fact,

$$
\begin{aligned}
& \left|u_{j+1}\left(t_{1}\right)-u_{j}\left(t_{1}\right)\right|-\left|u_{j+1}\left(t_{2}\right)-u_{j}\left(t_{2}\right)\right| \\
& \leq\left|u_{j+1}\left(t_{1}\right)-u_{j}\left(t_{1}\right)-u_{j+1}\left(t_{2}\right)+u_{j}\left(t_{2}\right)\right| \\
& \leq \mid \int_{t_{0}}^{t_{1}}\left(f\left(s, u_{j}(s)\right)-f\left(s, u_{j-1}(s)\right)\right) \Delta s \\
& \quad-\int_{t_{0}}^{t_{2}}\left(f\left(s, u_{j}(s)\right)-f\left(s, u_{j-1}(s)\right)\right) \Delta s \mid \\
& \leq 2 M \int_{t_{1}}^{t_{2}} \Delta s \leq 2 M\left(t_{2}-t_{1}\right) .
\end{aligned}
$$

The right-hand side in inequality (29) is at most $y\left(t_{2}\right)+\epsilon+$ $2 M\left(t_{2}-t_{1}\right)$ for large $n$ if $\epsilon>0$ provided that $\left|t_{2}-t_{1}\right| \leq \epsilon / 2 M$. Since $\epsilon$ is arbitrary and $t_{1}, t_{2}$ can be interchangeable, we get

$$
\left|y\left(t_{1}\right)-y\left(t_{2}\right)\right| \leq 2 M\left(t_{2}-t_{1}\right) \text {. }
$$

This implies that $y$ is continuous on $\left[t_{0}, t_{0}+\eta\right]_{\mathbb{V}}$. Using condition (H2) and the definition of successive approximations, we obtain

$$
\left|u_{j+1}(t)-u_{j}(t)\right| \leq c \int_{t_{0}}^{t}\left|u_{j}(s)-u_{j-1}(s)\right|^{\alpha} \Delta s .
$$

The sequence $\left\{u_{n}\right\}$ is equicontinuous: that is, for each function $u_{n}$ and any $\epsilon>0, t_{1}, t_{2} \in\left[t_{0}, t_{0}+\eta\right]_{\mathbb{T}}$ if there exists $\tau=\epsilon / M$ such that $t_{2}-t_{1} \leq \tau$, then

$$
\begin{aligned}
\left|u_{n+1}\left(t_{1}\right)-u_{n+1}\left(t_{2}\right)\right| & =\left|\int_{t_{1}}^{t_{2}} f\left(s, u_{n}(s)\right) \Delta s\right| \\
& \leq \int_{t_{1}}^{t_{2}}\left|\tilde{f}\left(s, u_{n}(s)\right)\right| d s \\
& \leq M\left(t_{1}-t_{2}\right) \leq \epsilon .
\end{aligned}
$$

All of the Arzela-Ascoli Theorem conditions are fulfilled for the family $\left\{u_{j}\right\}$ in $C_{\mathrm{rd}}\left[t_{0}, t_{0}+\eta\right]_{\mathbb{T}}$. Hence, there exists a subsequence $\left\{u_{j_{k}}\right\}$ converging uniformly on $\left[t_{0}, t_{0}+\eta\right]_{\mathbb{T}}$ as $j_{k} \rightarrow \infty$.

Let us note

$$
m^{*}(t)=\lim _{k \rightarrow \infty}\left|u_{j_{k}}(t)-u_{j_{k-1}}(t)\right| .
$$

Further, if $\left\{\left|u_{j}-u_{j-1}\right|\right\} \rightarrow 0$ as $j \rightarrow \infty$, then the limit of any subsequence is the unique solution $u$ of (25). It follows that a selection of subsequences is unnecessary and that the entire sequence $\left\{u_{j}\right\}$ converges uniformly to $u$. For that, it suffices to show that $y \equiv 0$ which will lead to $m^{*}(t)$ being null.

Setting

$$
R(t)=\int_{t_{0}}^{t} y(s)^{\alpha} d s
$$

and by defining $\psi^{*}(t)=t^{-k} y(t)$, we show that $\lim _{t \rightarrow 0^{+}} \psi^{*}(t)=$ 0 .

We prove by absurdity that $\psi^{*} \equiv 0$. Assume that $\psi^{*}(t)>$ 0 at any point in $\left.] t_{0}, t_{0}+\eta\right]_{\mathbb{T}}$; then there exists $t_{1}$ such that $0<\bar{m}=\psi^{*}\left(t_{1}\right)=\max _{t \in\left[t_{0}, t_{0}+\eta\right]_{\mathbb{T}}} \psi^{*}(t)$. Hence, from condition (H1), we obtain

$$
\bar{m}=\psi\left(t_{1}\right)=t_{1}^{-k} y\left(t_{1}\right) \leq \bar{m} t_{1}<\bar{m} .
$$

We end up with a contradiction. So $\psi^{*} \equiv 0$. Therefore, the Picard iterates (25) converge uniformly to the unique solution $u$ of $(2)$ on $\left[t_{0}, t_{0}+\eta\right]_{\mathbb{T}}$.

3.3. Uniqueness Results for Fractional Order ODE. In this section, we denote $C_{p}\left(\left[t_{0}, t_{0}+a\right]_{\mathbb{T}}, \mathbb{R}\right)=\left\{u \mid u \in C\left(\left[t_{0}, t_{0}+\right.\right.\right.$ $\left.a]_{\mathbb{T}}, \mathbb{R}\right)$ and $\left.\left(t-t_{0}\right)^{1-q} u \in C\left(\left[t_{0}, t_{0}+a\right]_{\mathbb{T}}, \mathbb{R}\right)\right\}$.

Theorem 15 (Krasnoselskii-Krein conditions). Let $f(t, x)$ be continuous in $S_{0}$ and satisfying for all $(t, x),(t, \bar{x}) \in S_{0}$

(J1) $|f(t, x)-f(t, \bar{x})| \leq k r \Gamma(\alpha)\left|t-t_{0}\right|^{-\alpha}|x-\bar{x}|, t \neq t_{0}$,

(J2) $|f(t, x)-f(t, \bar{x})| \leq c|x-\bar{x}|^{\delta}$, where $c, r, k$ are positive constants such that $k>1, k r \leq \alpha$, and $k(1-\delta)<1$, and the real number $\delta$ is such that $0<\delta<1$. 
Then, the fractional order initial value problem (3) has at most one solution on $\left[t_{0}, t_{0}+a\right]_{\mathbb{T}}$.

Proof. Suppose $u$ and $v$ are two solutions of (3) in $\left[t_{0}, t_{0}+a\right]_{\mathbb{V}}$. We will show that $u \equiv v$. Let us define $\phi(t)$ and $R(t)$ by

$$
\begin{aligned}
& \phi(t)=|u(t)-v(t)|, \quad \text { for every } t \in\left[t_{0}, t_{0}+a\right]_{\mathbb{T}}, \\
& R(t)=\frac{c}{\Gamma(\alpha)} \int_{t_{0}}^{t}(t-s)^{\alpha-1} \widetilde{\phi}^{\delta}(s) d s,
\end{aligned}
$$

for every $t \in\left[t_{0}, t_{0}+a\right]$,

such that $\widetilde{\phi}$ is the extension of $\phi$ to the real interval $\left[t_{0}, t_{0}+a\right]$. It follows from condition (J2) that

$$
\begin{aligned}
& \phi(t) \\
& \quad=\left|\frac{1}{\Gamma(\alpha)} \int_{t_{0}}^{t}(t-s)^{\alpha-1}[f(s, u(s))-f(s, v(s))] \Delta s\right| \\
& \quad \leq \frac{1}{\Gamma(\alpha)} \int_{t_{0}}^{t}(t-s)^{\alpha-1}|f(s, u(s))-f(s, v(s))| \Delta s \\
& \leq \frac{1}{\Gamma(\alpha)} \int_{t_{0}}^{t} c(t-s)^{\alpha-1}|u(s)-v(s)|^{\delta} \Delta s \\
& \quad \leq \int_{t_{0}}^{t} \frac{c}{\Gamma(\alpha)}(t-s)^{\alpha-1}|\tilde{u}(s)-\widetilde{v}(s)|^{\delta} d s=R(t) .
\end{aligned}
$$

On the other hand, $R\left(t_{0}\right)=0, R(t)>0$ for $t>t_{0}$, and ${ }_{t}^{\mathbb{T}} D^{\alpha} R(t)=\widetilde{\phi}^{\delta}(t)=R^{\delta}(t)$, for every $t \in\left[t_{0}, t_{0}+a\right]_{\mathbb{T}}$. Now from relations (37) and (38) and using Lemma 11, we obtain for every $t \in\left[t_{0}, t_{0}+a\right]_{\mathbb{T}}$

$$
\phi(t) \leq R(t)=L\left(t-t_{0}\right)^{\sigma},
$$

where $L$ and $\sigma$ are defined as in Lemma 11. Moreover, if we define $\psi(t)=\phi(t) /\left(t-t_{0}\right)^{k}$, we get

$$
0 \leq \psi(t) \leq L\left(t-t_{0}\right)^{\sigma-k \alpha},
$$

$$
\text { for every } t \in\left[t_{0}, t_{0}+a\right]_{\mathbb{V}} \text {. }
$$

It follows that the exponent of $t$ in the above inequality is positive, since $k(1-\delta)<1$. Hence, $\lim _{t \rightarrow t_{0}} \psi(t)=0$. Therefore, if we define $\psi\left(t_{0}\right)=0$, then the function is rd-continuous in $\left[t_{0}, t_{0}+a\right]_{\mathbb{T}}$.

Now, to show that $\phi \equiv 0$, we prove by absurdity that $\psi \equiv 0$ on $\left[t_{0}, t_{0}+a\right]_{\mathbb{T}}$. Assume that $\psi$ does not vanish at some points $t$; that is, $\psi(t)>0$ on $\left.] t_{0}, t_{0}+a\right]_{\pi}$; then there exists a maximum $m>0$ reached when $t$ is equal to some $t_{1}: t_{0}<t_{1} \leq t_{0}+a$ such that $\psi(s)<m=\psi\left(t_{1}\right)$, for $s \in\left[t_{0}, t_{1}\right)_{\mathbb{T}}$. But from condition (J1), we have

$$
\begin{aligned}
m & =\psi\left(t_{1}\right)=\left(t_{1}-t_{0}\right)^{-k} \phi\left(t_{1}\right)<\left(t_{1}-t_{0}\right)^{-k \alpha} \\
& \cdot \int_{t_{0}}^{t_{1}} k r(t-s)^{\alpha-1}[f(s, u(s))-f(s, v(s))] \Delta s \\
& \leq\left(t_{1}-t_{0}\right)^{-k \alpha} \int_{t_{0}}^{t_{1}} k r(t-s)^{\alpha-1} \frac{\phi(s)}{\left(s-t_{0}\right)^{-\alpha}} \Delta s
\end{aligned}
$$

$$
\begin{aligned}
& \leq\left(t_{1}-t_{0}\right)^{-k \alpha} \int_{t_{0}}^{t_{1}} k r(t-s)^{\alpha-1}\left(s-t_{0}\right)^{k \alpha-\alpha} \psi(s) \Delta s \\
& \leq m k r\left(t_{1}-t_{0}\right)^{-\alpha} \int_{t_{0}}^{t_{1}}\left(t_{1}-s\right)^{\alpha-1} \Delta s \\
& \leq m k r\left(t_{1}-t_{0}\right)^{-\alpha} \int_{t_{0}}^{t_{1}}\left(t_{1}-s\right)^{\alpha-1} d s \leq \frac{m k r}{\alpha}<m,
\end{aligned}
$$

which is a contradiction. Thus, the uniqueness of the solution is established.

Theorem 16 (Kooi's conditions). Let $f(t, x)$ be continuous in $S_{0}$ and satisfying for all $(t, x),(t, \bar{x}) \in S_{0}$

(K1) $|f(t, x)-f(t, \bar{x})| \leq k r \Gamma(\alpha)\left|t-t_{0}\right|^{-\alpha}|x-\bar{x}|, t \neq t_{0}$,

(K2) $\left|t-t_{0}\right|^{\beta}|f(t, x)-f(t, \bar{x})| \leq c|x-\bar{x}|^{\delta}$, where $c$, $r$, and $k$ are positive constants; the positive real numbers $\beta, \delta$, $k$, $r$ are such that $0<\beta<\delta<1$, and $k(1-\delta)<1-\beta$, and $k r \leq \alpha$.

Then, the first-order initial value problem (3) has at most one solution on $\left[t_{0}, t_{0}+a\right]_{\mathbb{T}}$.

Proof. The proof is similar to that of Theorem 15; thus, we omit it.

\subsection{Existence of Solutions under Krasnoselskii-Krein}

Conditions on Time Scales

Theorem 17. Assume that conditions (J1) and (J2) are satisfied; then the successive approximations given by

$$
\begin{gathered}
u_{n+1}(t)=\int_{t_{0}}^{t} f\left(s, u_{n}(s)\right) \Delta s \\
u_{0}(t)=0, \quad n=0,1, \ldots
\end{gathered}
$$

converge uniformly to the unique solution $u$ of (3) on $\left[t_{0}, t_{0}+\right.$ $\eta]_{\mathbb{T}}$, where

$$
\eta=\min \left\{a,\left(\frac{b \Gamma(1+\alpha)}{M}\right)^{1 / \alpha}\right\}
$$

and $M$ is the bound for $f$ on $S_{0}$.

Proof. With the uniqueness of the solution being proved in Theorem 15, we prove the existence of the solution using Arzela-Ascoli Theorem.

Step 1. The successive approximations $\left\{u_{n+1}\right\}, n=0,1,2, \ldots$ given by (42) are well defined and continuous. Indeed,

$$
\begin{aligned}
\left|u_{n+1}(t)\right| & =\left|\frac{1}{\Gamma(\alpha)} \int_{t_{0}}^{t}(t-s)^{\alpha-1} f\left(s, u_{n}(s)\right) \Delta s\right| \\
& \leq \frac{1}{\Gamma(\alpha)} \int_{t_{0}}^{t}(t-s)^{\alpha-1}\left|f\left(s, u_{n}(s)\right)\right| \Delta s .
\end{aligned}
$$


This yields, for $n=0$,

$$
\begin{aligned}
\left|u_{1}(t)\right| & \leq \frac{M}{\Gamma(\alpha)} \int_{t_{0}}^{t}(t-s)^{\alpha-1} \Delta s \\
& \leq \frac{M}{\Gamma(\alpha)} \int_{t_{0}}^{t}(t-s)^{\alpha-1} d s \leq \frac{M a^{\alpha}}{\Gamma(\alpha+1)} \leq b .
\end{aligned}
$$

By induction, the sequence $\left\{u_{j+1}(t)\right\}$ is well defined and uniformly bounded on $\left[t_{0}, t_{0}+\eta\right]_{\mathbb{T}}$.

Step 2. We prove that $y$ is a continuous function in $\left[t_{0}, t_{0}+\eta\right]_{\mathbb{T}}$, where $y$ is defined by

$$
y(t)=\limsup _{j \rightarrow \infty}\left|u_{j}(t)-u_{j-1}(t)\right| .
$$

For $t_{1}, t_{2} \in\left[t_{0}, t_{0}+\eta\right]_{\mathbb{T}}$, we have

$$
\begin{aligned}
\left|u_{j+1}\left(t_{1}\right)-u_{j}\left(t_{1}\right)\right| \leq & \left|u_{j+1}\left(t_{2}\right)-u_{j}\left(t_{2}\right)\right| \\
& +\frac{4 M}{\Gamma(\alpha+1)}\left(t_{2}-t_{1}\right)^{\alpha} .
\end{aligned}
$$

In fact,

$$
\begin{aligned}
& \left|u_{j+1}\left(t_{1}\right)-u_{j}\left(t_{1}\right)\right|-\left|u_{j+1}\left(t_{2}\right)-u_{j}\left(t_{2}\right)\right| \leq \mid u_{j+1}\left(t_{1}\right) \\
& -u_{j}\left(t_{1}\right)-u_{j+1}\left(t_{2}\right)+u_{j}\left(t_{2}\right) \\
& \leq \frac{1}{\Gamma(\alpha)} \mid \int_{t_{0}}^{t_{1}}\left(t_{1}-s\right)^{\alpha-1} \\
& \cdot\left(f\left(s, u_{j}(s)\right)-f\left(s, u_{j-1}(s)\right)\right) \Delta s \\
& -\int_{0}^{t_{2}}\left(t_{2}-s\right)^{\alpha-1} \\
& \cdot\left(f\left(s, u_{j}(s)\right)-f\left(s, u_{j-1}(s)\right)\right) \Delta s \mid \\
& \leq \frac{2 M}{\Gamma(\alpha)}\left[\mid \int_{0}^{t_{1}}\left(\left(t_{1}-s\right)^{\alpha-1}-\left(t_{2}-s\right)^{\alpha-1}\right) \Delta s\right. \\
& \left.-\int_{t_{1}}^{t_{2}}\left(t_{2}-s\right)^{\alpha-1} \Delta s \mid\right] \\
& \leq \frac{2 M}{\Gamma(\alpha)}\left[\mid \int_{0}^{t_{1}}\left(\left(t_{1}-s\right)^{\alpha-1}-\left(t_{2}-s\right)^{\alpha-1}\right) d s\right. \\
& \left.-\int_{t_{1}}^{t_{2}}\left(t_{2}-s\right)^{\alpha-1} d s \mid\right] \leq \frac{2 M}{\alpha \Gamma(\alpha)}\left[t_{1}^{\alpha}-t_{2}^{\alpha}+2\left(t_{2}\right.\right. \\
& \left.\left.-t_{1}\right)^{\alpha}\right] \leq \frac{4 M}{\Gamma(\alpha+1)}\left(t_{2}-t_{1}\right)^{\alpha}
\end{aligned}
$$

The right-hand side in inequality (47) is at most $y\left(t_{2}\right)+\epsilon+$ $(4 M / \Gamma(\alpha+1))\left(t_{2}-t_{1}\right)^{\alpha}$ for large $n$ if $\epsilon>0$ provided that $\left|t_{2}-t_{1}\right| \leq(\epsilon \Gamma(\alpha+1) / 4 M)^{1 / \alpha}$. Since $\epsilon$ is arbitrary and $t_{1}, t_{2}$ can be interchangeable, we get

$$
\left|y\left(t_{1}\right)-y\left(t_{2}\right)\right| \leq \frac{4 M}{\Gamma(\alpha+1)}\left(t_{2}-t_{1}\right) .
$$

This implies that $y$ is continuous on $\left[t_{0}, t_{0}+\eta\right]_{\mathbb{T}}$. Using condition (J2) and the definition of successive approximations, we obtain

$$
\left|u_{j+1}(t)-u_{j}(t)\right| \leq \frac{c}{\Gamma(\alpha)} \int_{t_{0}}^{t}\left[\left|u_{j}(s)-u_{j-1}(s)\right|^{\alpha}\right] \Delta s .
$$

The sequence $\left\{u_{n}\right\}$ is equicontinuous: that is, for each function $u_{n}$ and any $\epsilon>0, t_{1}, t_{2} \in\left[t_{0}, t_{0}+\eta\right]_{\mathbb{T}}$ if there exists $\tau=$ $\epsilon^{-\alpha} \Gamma(\alpha+1) / M$ such that $t_{2}-t_{1} \leq \tau$; then

$$
\begin{aligned}
& \left|u_{n+1}\left(t_{1}\right)-u_{n+1}\left(t_{2}\right)\right| \\
& \quad=\mid \frac{1}{\Gamma(\alpha)} \int_{t_{0}}^{t_{2}}\left(t_{2}-s\right)^{\alpha-1} f\left(s, u_{n}(s)\right) \Delta s \\
& \quad-\int_{t_{0}}^{t_{1}}\left(t_{1}-s\right)^{\alpha-1} f\left(s, u_{n}(s)\right) \Delta s \mid \leq \frac{2 M}{\Gamma(\alpha+1)}\left(t_{1}\right. \\
& \left.-t_{2}\right)^{\alpha} \leq \epsilon,
\end{aligned}
$$

where we used a similar argument as in (48).

All of the Arzela-Ascoli Theorem conditions are fulfilled for the family $\left\{u_{j}\right\}$ in $C_{\mathrm{rd}}\left[t_{0}, t_{0}+a\right]_{\mathbb{T}}$. Hence, there exists a subsequence $\left\{u_{j_{k}}\right\}$ converging uniformly on $\left[t_{0}, t_{0}+a\right]_{\mathbb{T}}$ as $j_{k} \rightarrow \infty$.

Let us note

$$
m^{*}(t)=\lim _{k \rightarrow \infty}\left|u_{j_{k}}(t)-u_{j_{k-1}}(t)\right| .
$$

Further, if $\left\{\left|u_{j}-u_{j-1}\right|\right\} \rightarrow 0$ as $j \rightarrow \infty$, then the limit of any subsequence is the unique solution $u$ of (42). It follows that a selection of subsequences is unnecessary and that the entire sequence $\left\{u_{j}\right\}$ converges uniformly to $u$. For that, it is sufficient to show that $y \equiv 0$ which will lead to $m^{*}(t)$ being null.

Setting

$$
R(t)=\frac{c}{\Gamma(\alpha)} \int_{t_{0}}^{t}(t-s)^{\alpha-1} y(s)^{\alpha} d s,
$$

and defining $\psi^{*}(t)=t^{-k} y(t)$ and then using Lemma 11, we obtain that $\psi(t) \leq L\left(t_{1}-t_{0}\right)^{J-k \alpha}$. Which yields that $\lim _{t \rightarrow 0^{+}} \psi^{*}(t)=0$.

We prove by absurdity that $\psi^{*} \equiv 0$. Assume that $\psi^{*}(t)>$ 0 at any point in $\left.] t_{0}, t_{0}+\eta\right]_{\mathbb{T}}$; then there exists $t_{1}$ such that $0<\bar{m}=\psi^{*}\left(t_{1}\right)=\max _{t \in\left[t_{0}, t_{0}+\eta\right]_{\pi}} \psi^{*}(t)$. Hence, from condition (J1), we obtain

$$
\begin{aligned}
m & =\psi\left(t_{1}\right)=\left(t_{1}-t_{0}\right)^{-k \alpha} \phi\left(t_{1}\right) \\
& \leq\left(t_{1}-t_{0}\right)^{-k \alpha} \int_{t_{0}}^{t_{1}} k r\left(t_{1}-s\right)^{\alpha-1}\left(s-t_{0}\right)^{-\alpha} \phi(s) d s \\
& \leq k r\left(t_{1}-t_{0}\right)^{-k \alpha} \int_{t_{0}}^{t_{1}}\left(t_{1}-s\right)^{\alpha-1}\left(s-t_{0}\right)^{k \alpha-\alpha} \psi(s) d s \\
& <k r m\left(t_{1}-t_{0}\right)^{-\alpha} \int_{t_{0}}^{t_{1}}\left(t_{1}-t_{0}\right)^{\alpha-1} d s<\frac{k r m}{\alpha}<m .
\end{aligned}
$$

We end up with a contradiction. So $\psi^{*} \equiv 0$. Therefore, the Picard iterates (42) converge uniformly to the unique solution $u$ of $(2)$ on $\left[t_{0}, t_{0}+\eta\right]_{\mathbb{T}}$. 
Remark 18. For the case $\mathbb{T}=\mathbb{R}$, Theorem 15 is reduced to $[31$, Theorem 2.1].

\section{Competing Interests}

The authors declare that there is no conflict of interests regarding the publication of this paper.

\section{References}

[1] R. P. Agarwal and M. Bohner, "Basic calculus on time scales and some of its applications," Results in Mathematics, vol. 35, no. 1-2, pp. 3-22, 1999.

[2] M. Bohner and A. Peterson, Dynamic Equations on Time Scales, Birkhäuser, Boston, Mass, USA, 2001.

[3] M. Bohner and A. Peterson, Eds., Advances in Dynamic Equations on Time Scales, Birkhäuser, Boston, Mass, USA, 2003.

[4] G. S. Guseinov, "Integration on time scales," Journal of Mathematical Analysis and Applications, vol. 285, no. 1, pp. 107-127, 2003.

[5] G. S. Guseinov and B. Kaymakçalan, "Basics of Riemann delta and nabla integration on time scales," Journal of Difference Equations and Applications, vol. 8, no. 11, pp. 1001-1017, 2002.

[6] R. J. Higgins, Oscillation theory of dynamic equations on time scales [Ph.D. thesis], University of Nebraska-Lincoln, Lincoln, Neb, USA, 2008, ProQuest LLC, Ann Arbor, MI, 2008.

[7] S. Hilger, "Analysis on measure chains-a unified approach to continuous and discrete calculus," Results in Mathematics, vol. 18, no. 1-2, pp. 18-56, 1990.

[8] C. C. Tisdell and A. Zaidi, "Basic qualitative and quantitative results for solutions to nonlinear, dynamic equations on time scales with an application to economic modelling," Nonlinear Analysis: Theory, Methods \& Applications, vol. 68, no. 11, pp. 3504-3524, 2008.

[9] R. Agarwal, M. Bohner, D. O’Regan, and A. Peterson, "Dynamic equations on time scales: a survey," Journal of Computational and Applied Mathematics, vol. 141, no. 1-2, pp. 1-26, 2002.

[10] N. Benkhettou, A. Hammoudi, and D. F. M. Torres, "Existence and uniqueness of solution for a fractional riemann-liouville initial value problem on time scales," Journal of King Saud University-Science, vol. 28, no. 1, pp. 87-92, 2016.

[11] M. Bohner and A. Peterson, "First and second order linear dynamic equations on time scales," Journal of Difference Equations and Applications, vol. 7, no. 6, pp. 767-792, 2001.

[12] A. Chidouh, A. Guezane-Lakoud, and R. Bebbouchi, "Positive solutions for an oscillator fractional initial value problem," Journal of Applied Mathematics and Computing, 2016.

[13] A. Chidouh, A. Guezane-Lakoud, and R. Bebbouchi, "Positive solutions of the fractional relaxation equation using lower and upper solutions," Vietnam Journal of Mathematics, 2016.

[14] J. M. Gallardo, "Second-order differential operators with integral boundary conditions and generation of analytic semigroups," The Rocky Mountain Journal of Mathematics, vol. 30, no. 4, pp. 1265-1291, 2000.

[15] A. Guezane-Lakoud, "Initial value problem of fractional order," Cogent Mathematics, vol. 2, no. 1, Article ID 1004797, 2015.

[16] A. Guezane-Lakoud and R. Khaldi, "Solvability of a fractional boundary value problem with fractional integral condition," Nonlinear Analysis: Theory, Methods \& Applications, vol. 75, no. 4, pp. 2692-2700, 2012.
[17] A. Guezane-Lakoud and R. Khaldi, "Solvability of a three-point fractional nonlinear boundary value problem," Differential Equations and Dynamical Systems, vol. 20, no. 4, pp. 395-403, 2012.

[18] A. Guezane-Lakoud and A. Kiliçman, "Unbounded solution for a fractional boundary value problem," Advances in Difference Equations, vol. 2014, article 154, 2014.

[19] A. Souahi, A. Guezane-Lakoud, and A. Hitta, "On the existence and uniqueness for high order fuzzy fractional differential equations with uncertainty," Advances in Fuzzy Systems, vol. 2016, Article ID 5246430, 9 pages, 2016.

[20] A. H. Zaidi, "Existence of solutions and convergence results for dynamic initial value problems using lower and upper solutions," Electronic Journal of Differential Equations, No. 161, 13 pages, 2009.

[21] D. R. Anderson and A. Boucherif, "Nonlocal initial value problem for first-order dynamic equations on time scales," Discrete and Continuous Dynamical Systems, Series A: Mathematical Analysis, vol. 16, supplement S1, pp. 222-226, 2009.

[22] Q. Dai and C. C. Tisdell, "Existence of solutions to first-order dynamic boundary value problems," International Journal of Difference Equations, vol. 1, no. 1, pp. 1-17, 2006.

[23] I. L. dos Santos, "On qualitative and quantitative results for solutions to first-order dynamic equations on time scales," Boletín de la Sociedad Matemática Mexicana, vol. 21, no. 2, pp. 205-218, 2015.

[24] H. Gilbert, "Existence theorems for first-order equations on time scales with $\Delta$-Carathéodory functions," Advances in Difference Equations, vol. 2010, Article ID 650827, 2010.

[25] C. C. Tisdell, "On first-order discrete boundary value problems," Journal of Difference Equations and Applications, vol. 12, no. 12, pp. 1213-1223, 2006.

[26] R. P. Agarwal and V. Lakshmikantham, Uniqueness and Nonuniqueness Criteria for Ordinary Differential Equations, vol. 6 of Series in Real Analysis, World Scientific Publishing, River Edge, NJ, USA, 1993.

[27] A. Anguraj and P. Karthikeyan, "Existence of solutions for nonlocal semilinear fractional integro-differential equation with Krasnoselskii-KREin-type conditions," Nonlinear Studies, vol. 19, no. 3, pp. 433-442, 2012.

[28] F. M. Atici, A. Cabada, C. J. Chyan, and B. Kaymakçalan, "Nagumo type existence results for second-order nonlinear dynamic BVPs," Nonlinear Analysis: Theory, Methods \& Applications, vol. 60, no. 2, pp. 209-220, 2005.

[29] R. A. C. Ferreira, "A Nagumo-type uniqueness result for an nth order differential equation," Bulletin of the London Mathematical Society, vol. 45, no. 5, pp. 930-934, 2013.

[30] M. A. Krasnosel'skiǐ and S. G. Krein, "On a class of uniqueness theorems for the equation $y=f(x, y)$," Uspekhi Matematicheskikh Nauk, vol. 11, no. 1(67), pp. 209-213, 1956.

[31] V. Lakshmikantham and S. Leela, "A Krasnoselskii-KREintype uniqueness result for fractional differential equations," Nonlinear Analysis: Theory, Methods \& Applications, vol. 71, no. 7-8, pp. 3421-3424, 2009.

[32] V. Lakshmikantham and S. Leela, "Nagumo-type uniqueness result for fractional differential equations," Nonlinear Analysis: Theory, Methods \& Applications, vol. 71, no. 7-8, pp. 2886-2889, 2009.

[33] O. G. Mustafa and D. O’Regan, "On the Nagumo uniqueness theorem," Nonlinear Analysis: Theory, Methods \& Applications, vol. 74, no. 17, pp. 6383-6386, 2011. 
[34] F. Yoruk, T. G. Bhaskar, and R. P. Agarwal, "New uniqueness results for fractional differential equations," Applicable Analysis, vol. 92, no. 2, pp. 259-269, 2013.

[35] S. G. Samko, A. A. Kilbas, and O. I. Marichev, Fractional Integrals and Derivatives, Gordon and Breach Science Publishers, Yverdon, Switzerland, 1993, Theory and Applications, Edited and with a foreword by S. M. Nikol'skiü, Translated from the 1987 Russian Original. 


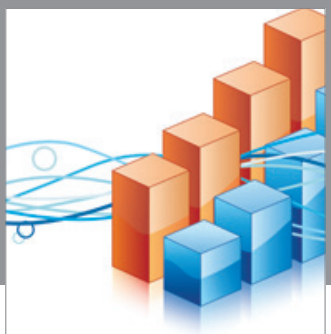

Advances in

Operations Research

vatem alat4

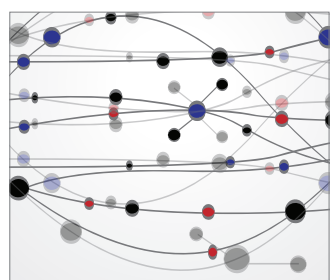

\section{The Scientific} World Journal
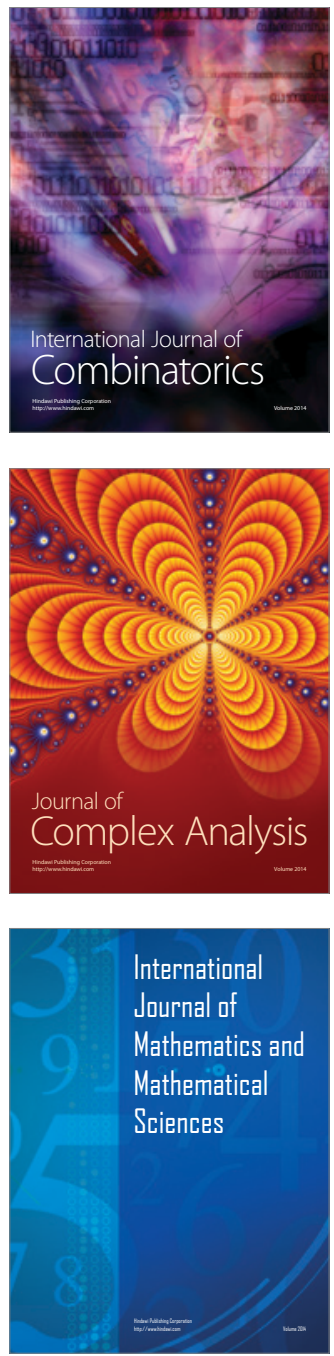
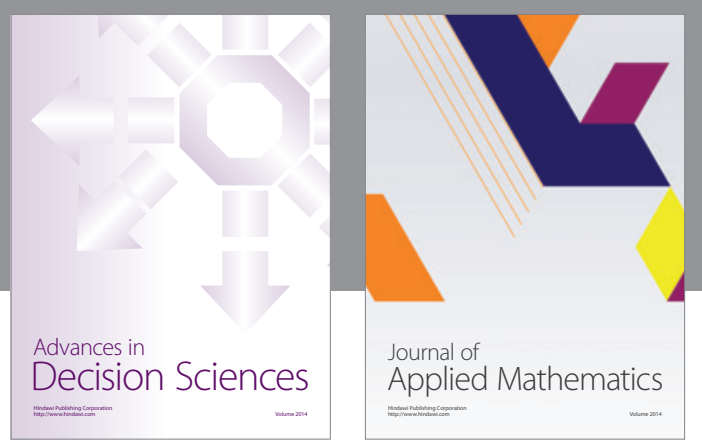

Algebra

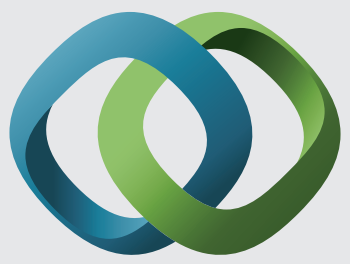

\section{Hindawi}

Submit your manuscripts at

http://www.hindawi.com
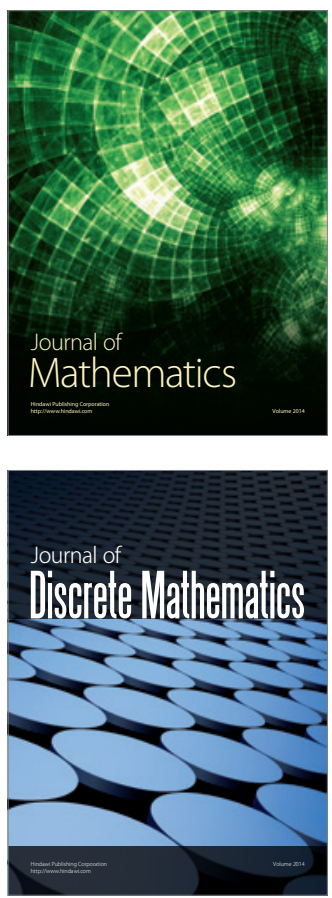

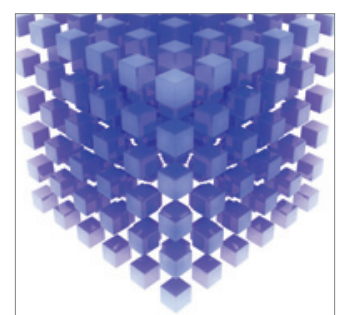

Mathematical Problems in Engineering
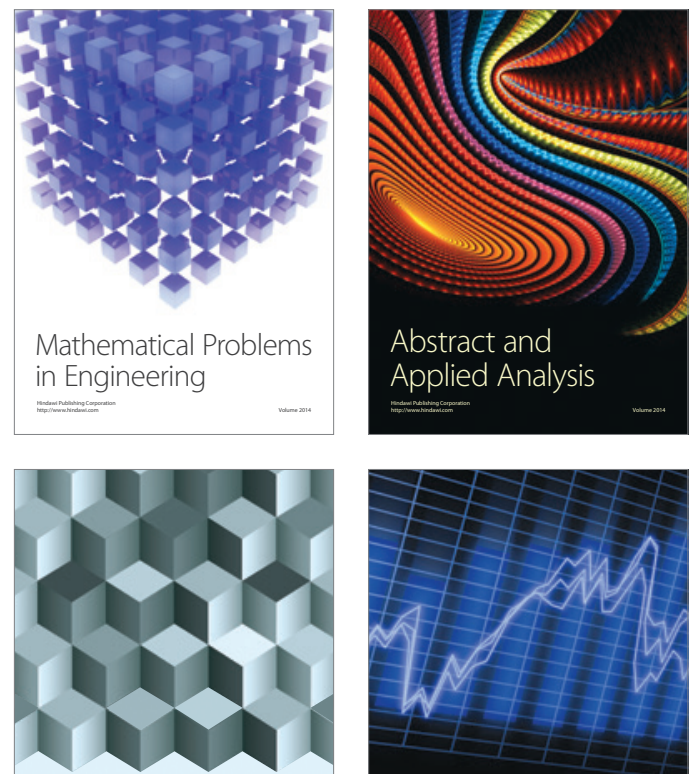

Journal of

Function Spaces

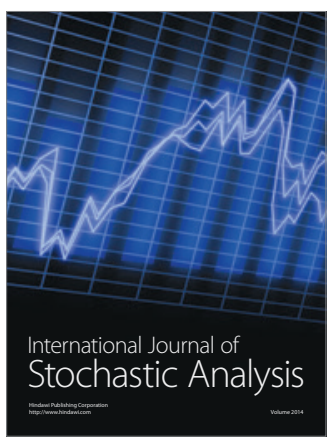

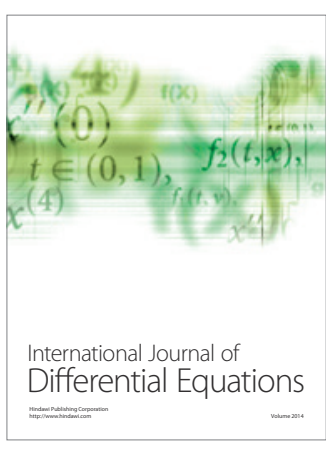
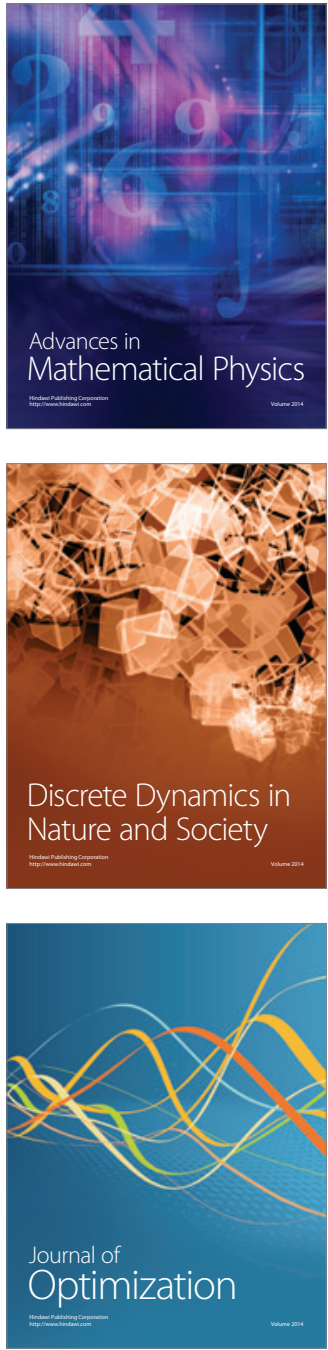